\title{
Treatment guidelines for depression in pregnancy
}

\author{
S.M. Marcus ${ }^{\mathrm{a}, *}$, K.L. Barry ${ }^{\mathrm{b}, \mathrm{c}}$, H.A. Flynn ${ }^{\mathrm{b}}$, R. Tandon ${ }^{\mathrm{d}}$, J.F. Greden ${ }^{\mathrm{b}, \mathrm{e}}$ \\ ${ }^{a}$ University of Michigan Department of Psychiatry, Ambulatory Services and Women's Health Program, Ann Arbor, MI, USA \\ ${ }^{\mathrm{b}}$ University of Michigan Department of Psychiatry, Ann Arbor, MI, USA \\ ${ }^{\mathrm{c}}$ Department of Veterans Affairs, Serious Mental Illness Treatment Research and Evaluation Center, Ann Arbor, MI, USA \\ ${ }^{\mathrm{d}}$ Hospital Services, University of Michigan Department of Psychiatry, Ann Arbor, MI, USA \\ ${ }^{\mathrm{e}}$ Mental Health Research Institute, Ann Arbor, MI, USA
}

Received 24 May 2000; accepted 4 June 2000

\begin{abstract}
Depression is a ubiquitous disorder in childbearing women with up to $10 \%$ of women experiencing depression in pregnancy. Postpartum depression occurs in $12-16 \%$ of pregnancies making it a common complication. Moreover, these illnesses are frequently underdiagnosed in obstetric settings, and a recent report of the Surgeon General's Office confirms that many women do not access services, or receive treatment of inadequate intensity or duration. This paper provides current treatment guidelines to aid in appropriate diagnosis and treatment of depression in pregnancy and postpartum. Review of current literature on psychotropic medication use in pregnancy is also provided. (c) 2001 International Federation of Gynecology and Obstetrics. All rights reserved.
\end{abstract}

Keywords: Pregnancy; Postpartum; Depression; Antidepressant medication

\section{Introduction}

Depression is among the most common of health disorders in women. There are five key factors that are important to further characterize predictors of Major Depressive Disorder (MDD) during pregnancy in order to develop more effec-

\footnotetext{
* Corresponding author. Adult Ambulatory Division and Depression Center, University of Michigan Health Systems, Ann Arbor, MI, USA. Tel.: +1-734-764-0267.

E-mail address: smmarcus@umich.edu (S.M. Marcus).
}

tive strategies for treatment and prevention. First, almost one woman in four will experience depression at some point in her life [1]. Second, depressive episodes present most often during the childbearing years, and almost $10 \%$ of women will experience depression during pregnancy [2]. Third, MDD is now recognized as a recurrent disorder, with risks of relapse increasing for those with prior episodes following antidepressant discontinuation. Fourth, it is commonplace for physicians to withdraw antidepressant medications at the time of conception for women receiving treat- 
ment for depression. Fifth, there is little information available about specific risk factors for relapse during pregnancy, or strategies for preventing episodes. It is critical for clinicians treating childbearing women to have information available to guide them in treatment decisions as they negotiate the delicate balance between use of psychotropic medication during pregnancy vs. the risks of undertreating the woman's depressive illness and risking relapse. The purpose of this paper is to review the available data on the risks and sequelae of using various psychotropic medications during pregnancy and lactation, and to provide guidelines for the treatment of major mood disorders through pregnancy and postpartum. These guidelines will emphasize pharmacologic, psychotherapeutic, and psychosocial management strategies.

\subsection{Background}

Although there have been advances in psychopharmacologic treatment for depression, a recent report of the United States Surgeon General's Office confirms that many women do not seek treatment. Many who do seek treatment for depression do so in the primary care setting where $50 \%$ go unrecognized and many more are undertreated [3]. At the University of Michigan Health System, pilot data suggests that a diagnosis of depression is made in only $0.8 \%$ of childbearing women [4], however, national data suggests $10-20 \%$ prevalence of the disorder in medical/ surgical settings [5]. Of those who are diagnosed, most receive inadequate treatments. In addition, medical adherence is extremely problematic; half of antidepressants prescribed are not filled after the first month [6]. The under-detection and under-treatment of depression in women of childbearing age leads to substantial maternal and infant morbidity, and medical costs. Psychiatric illness has adverse consequences in pregnancy including inadequate nutrition, poor compliance with prenatal care, poor pregnancy outcomes, substance abuse, and dangerous risk-taking behavior [7]. The more dramatic outcomes such as risk taking, fetal abuse, or maternal suicide may be the disastrous consequences of untreated depression in pregnancy [8]. In women with bipolar illness, the risk of postpartum relapse is well established and estimated between 30 and $50 \%$ particularly when mood stabilizers are discontinued [9].

Due to the prevalence of psychiatric disorders in childbearing women, many women are using psychotropic medications at the time of conception. As all psychoactive medications cross the placental barrier, the woman's health and disease management must be balanced against the risk of infant exposure during pregnancy. Many times the risk of medication is outweighed by the substantial morbidity of the undertreated illness.

\section{Research on adverse effects of psychotropic medications in pregnancy}

Psychotropic medications potentially can have three types of adverse effects on the fetus: (1) teratogenic effects increase risk of congenital malformation usually during the first trimester; (2) neonatal toxicity and withdrawal syndromes are usually related to ongoing third trimester exposure; (3) behavioral effects are more difficult to characterize and are manifest by later behavioral and developmental difficulties in the child.

The FDA has assigned risk categories B, C, D, and $\mathrm{X}$ to different psychotropic medications. Category A indicates no risk. The risk categories are useful even though estimates of the teratogenic effects of various psychotropic medications are imprecise. Bupropion, clozapine, and buspirone have received the $\mathrm{B}$ designation, which indicates an absence of human risk with the caveat of limited data. SSRIs (fluoxetine, sertraline, paroxetine), desipramine, venlafaxine, mirtazapine, nefazodone, clonazepam, and most conventional and atypical antipsychotics have received the $\mathrm{C}$ designation. This denotes that human risk cannot be entirely eliminated because of inadequate human clinical trials and either no animal studies or, in some, evidence of risk in animals. The D designation has been assigned to lithium, carbamazepine, sodium valproate, most tricyclic antidepressants, and other benzodiazepines (other than clonazepam), indicating evidence of fetal risk but not 
an across the board contraindication during pregnancy. Benzodiazepines, including triazolam, temazepam, flurazepam, and quazepam have received the $\mathrm{X}$ (completely contraindicated) designation.

\section{Antidepressant and mood stabilizing agents}

There are significant methodological issues in the study of antidepressant medications in pregnancy. Factors including the use of other medications, the impact of other comorbid illnesses and use of alcohol and nicotine, genetic factors, maternal age, and time of gestation can confound the results of studies in this area [10]. Table 1 includes the teratogenicity, neonatal toxicity, and behavioral risks associated with antidepressant and mood stabilizing agents during pregnancy and lactation. This section briefly summarizes the most salient studies for each type of agent.

\subsection{Selective serotonin reuptake inhibitors}

\subsubsection{Pregnancy}

Recent research on the use of SSRIs (fluoxetine, sertraline, paroxetine, fluvoxamine) suggests that they are relatively safe for both the mother and fetus during pregnancy. Studies with fluvoxamine, paroxetine and sertraline have failed to show an increased risk of fetal anomalies [11,12]. There have been no maternal reports of adverse sequelae in the infants exposed to sertraline [13]. Moreover, longer term studies assessing infant development reveal that infants exposed to a variety of tricyclic agents as well as fluoxetine were developmentally normal and similar to sibling controls on measures of IQ and learning at the age of 5 years [14]. In another European study, no increase in congenital abnormalities was found in the perinatal period in 969 infants whose mothers used SSRIs and/or tricyclic antidepressants [15]. The study was unique in that it included 375 infants exposed to citalopram, a newly approved SSRI in the United States. These findings did not support two adverse reaction reports linking citalopram during early pregnancy with optic nerve hypoplasia and septum pellucidum defects.

Not all studies have supported the safety of the SSRIs in pregnancy and one group [16] concluded from a prospective cohort study that the infants of women who take fluoxetine in the first trimester are at increased risk for minor anomalies, such as larger for their gestational age with $55 \%$ falling into the 90th percentile and above for birthweight indicating a teratogenic effect. They also reported that those exposed during the third trimester are at increased risk for premature delivery, poor neonatal adaptation, cyanosis on feeding, and jitteriness. The findings of this study have been criticized for confounding factors of severity of depressive illness in the mother and increased maternal age [17].

\subsubsection{Lactation}

Fluoxetine and sertraline are secreted in breast milk and there is one report of infant irritability with exposure to fluoxetine through lactation, though no reports of neonatal difficulties in lactating infants who's mothers used sertraline [12]. Paroxetine was not found in measurable quantities in breast milk, and there were not reports of infant difficulties when this agent was used.

\subsubsection{General information}

SSRIs are excellent first line agents and can also be used with panic disorder or obsessive compulsive disorder. Potential side effects fluoxetine can include agitation, insomnia, gastrointestinal distress such as nausea or diarrhea, facial tension or headaches, sexual side effects, or weight gain.

\subsection{Tricyclic antidepressants}

\subsubsection{Pregnancy}

There are many studies exploring the risk of fetal exposure to the tricyclic agents during pregnancy. A comprehensive review of the literature from 1966 to 1995 did not reveal an association between fetal exposure to tricyclic antidepres- 


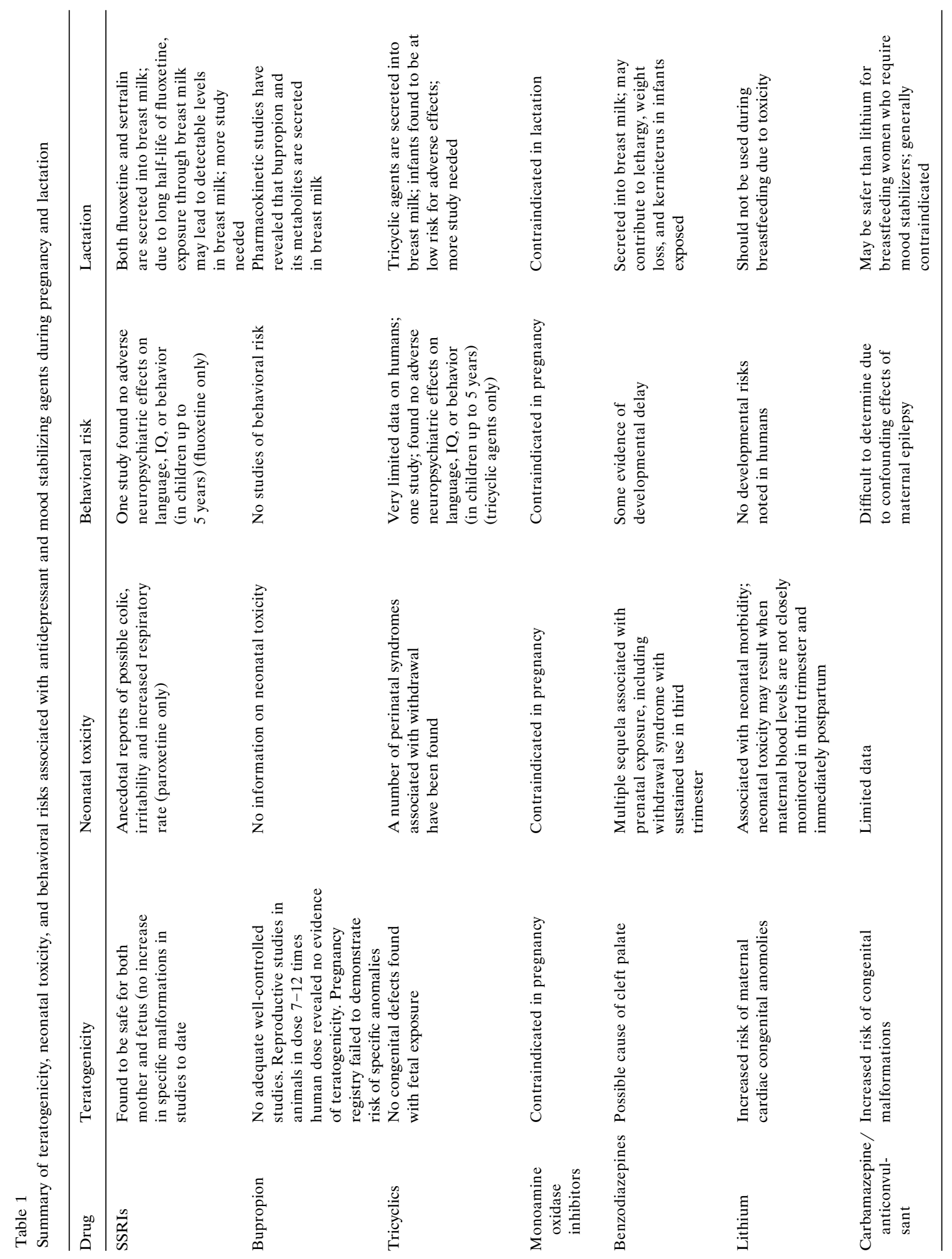


sants and rates or clustering of congenital malformation in 414 cases of first trimester exposure [9].

\subsubsection{Lactation}

Long-term follow-up studies on developmental outcomes of infants exposed to tricyclics through lactation are limited. These agents are secreted in breast milk, with a serum to milk ratio approximately equal to 1 . Levels in infant serum are variable and sometimes undetectable. Despite a limited database, most reviews support the use of secondary amine tricyclics (nortriptyline and desipramine) as first line agents for treatment of depression in nursing women [12].

\subsubsection{General information}

Tricyclics are good agents for patients with proven response but they have more side effects than SSRIs. Side effects include dry mouth, blurred vision, constipation, dizziness, cardiac symptoms, sedation or agitation, and weight gain. These agents can be lethal in an overdose.

\subsection{Other antidepressant agents}

Monoamine oxidase inhibitors have been associated with growth retardation, congenital anomalies and fetal death in animal and human studies and are contraindicated in pregnancy. Additionally, there are no published controlled clinical trials involving bupropion, venlafaxine, trazodone, or mirtazapine during pregnancy or lactation. Animal studies with bupropion have revealed no evidence of impaired fertility or fetal harm and retrospective analysis in the bupropion pregnancy registry did not show increased fetal anomalies [18].

\subsection{Alternative therapies and the nutraceuticals}

European double blind placebo controlled studies involving St John's Wort (Hypericum perforatum) have demonstrated efficacy in treating mild-moderate depression but the potency of the psychoactive substance and contaminants in the various preparations confound these studies. More research is needed in this area. Because some women self-medicate with herbal remedies, assuming them to be 'natural' and fail to consider potential toxicity in pregnancy or lactation, it is important for clinicians to inquire about herbal remedies when obtaining clinical histories in childbearing women.

\subsection{Benzodiazepines}

The early literature on benzodiazepines suggests that they contribute to cleft palate during first trimester exposure though the degree of risk is difficult to determine. The risk of cleft palate in the general population is approximately $0.064 \%$. In women using benzodiazepines during the first trimester, the risk appears to be increased, but still less than $1 \%$ in infants of women who are exposed [9]. There is evidence for adverse neonatal syndromes associated with consistent late third trimester exposure to benzodiazepines, including 'floppy infant syndrome' with hypotonia, lethargy, and poor temperature regulation.

\subsection{Mood stabilizers}

Lithium has been well studied in pregnancy and general cardiac anomalies occur in approximately $7 \%$ of bipolar women taking lithium. The degree of risk of the most common cardiac anomaly, Epstein's Anomaly, is estimated at $0.1 \%$ or 10-20 times the rate in the general population. This is much lower than initial estimates from the International Register of Lithium Babies [19]. Lithium is associated with neonatal morbidity and toxicity in the neonate and may result from nontoxic maternal levels. Clinical manifestations include hypotonia, hypothyroidism, cyanosis, lethargy, poor sucking, and abnormal reflexes.

Prenatal exposure to valproic acid has been associated with a $1-5 \%$ risk of neural tube defects. The incidence of neural tube anomalies in carbamazepine exposed children is $0.5 \%$ and other anomalies such as hypoplastic nails and craniofacial anomalies have also been identified [9]. Risk may be increased with the use of multiple anticonvulsant drugs or higher maternal plasma levels. The data on behavioral teratogenicity related to anticonvulsant exposure is difficult to interpret because the results are complicated by factors 
such as parental IQ, impact of seizure disorders in pregnancy, maternal blood levels of anticonvulsants, and timing of exposure [9]. A prospective controlled trial demonstrated no adverse neurobehavioral outcome in exposed children as indicated by IQ and language development [20]. Carbamazepine is metabolized more rapidly neonatally than lithium and may be a safer agent for nursing mothers.

\section{Management of depression in pregnancy}

Depression that is left untreated or not properly treated has been shown to negatively impact the health and overall functioning of women during pregnancy and in the postpartum. The following treatment guidelines are provided to assist with proper treatment in this population.

\subsection{Step $1-$ making a diagnosis}

Establishing a diagnosis of depression is a critical first step in its treatment. Frequent tearfulness, irritability or complaints of insomnia are clues that depression screening is necessary. Patients who present with features of depression for a period of two weeks or greater and who have impaired psychosocial functioning warrant a diagnosis of major depressive disorder (see Table 2). Many women are not forthcoming about their symptoms and may present with somatic manifestations of their illness. Sleep problems, headache, fatigue, gastrointestinal distress, or anxiety are common complaints. A comprehensive evaluation should include thyroid screening, as well as assessment for alcohol abuse. Many women who are depressed may use alcohol to self-medicate their symptoms. Other medications including progesterone containing compounds, cardiac medications, steroids, antihistamines, analgesics, and anticholingergics may induce depressive symptoms.

\subsection{Step 2 - determine severity of illness / period of interepisode wellbeing}

Many women prefer to remain medication free during their pregnancy if their symptoms are not severe. Patients with only mild impairment of mood, in whom sleep and appetite are unimpaired, and who have reasonable daytime functioning and no suicidal ideation would be candidates for non-pharmacologic management strategies. Likewise, childbearing women taking antidepressant agents at the time of conception may discontinue them, assuming they are asymptomatic, have a long period of inter-episode well-

Table 2

Diagnostic and Statistical Manual of Mental Disorders - IV Diagnostic [21] criteria for Major Depressive Episode

Five or more of the following symptoms that have been present for the same 2-week period or longer lat least one symptoms is either: (1) depressed mood or (2) loss of interest or pleasure]:

Depressed mood most of the day nearly every day

Diminished interest or pleasure in almost all activities most of the day nearly every day

Significant weight loss (more than $5 \%$ body wt.) when not dieting or significant change in appetite nearly every day

Insomnia or hypersomnia nearly every day

Psychomotor agitation or retardation nearly every day

Fatigue or loss of energy nearly every day

Feelings of worthlessness or excessive or inappropriate guilt nearly every day

Diminished ability to think or concentrate or indecisiveness nearly every day

Recurrent thoughts of death, recurrent suicidal ideation without a specific plan, or a suicide attempt or specific plan

for committing suicide

The symptoms cause clinically significant distress or impairment in social, occupational or other

important areas of functioning

The symptoms are not due to the direct physiological effects of a substance or medical condition

The symptoms are not better accounted for by bereavement 
being, and a history of mild illness. Prepregnancy planning is important in this population so that decisions can be made regarding pharmacotherapy. Medications should be weaned, as precipitous discontinuation may predispose to relapse. Women who are medication free should be carefully followed for recurrence or exacerbation of symptoms during their antenatal visits.

\subsection{Step 3 - initiating prevention strategies for women with mild illness}

Many therapeutic prevention strategies may be employed to prevent the exacerbation of minor symptoms, including a healthy diet free of alcohol, nicotine, and caffeine. Emphasizing proper sleep hygiene, stress management, and exercise are helpful measures. Mobilizing support systems may also be helpful in preventing relapse in women who are single or geographically distant from extended family. Individual or conjoint treatment is a very helpful adjunctive strategy especially when psychosocial stressors exist. Referral for cognitive behavioral therapy, or interpersonal therapy also reduces relapse risk in vulnerable populations of women with past history of major depression. Close monitoring during antenatal checks is important, and clinicians should proceed with pharmacotherapy, if symptoms become more severe.

\subsection{Step $4-$ pharmacological treatments of major depression}

The psychosocial management strategies described above should always be used as adjunctive strategies even in women with more severe illness. Women who have moderate to severe illness impeding their ability to care for themselves nutritionally or medically, or who are predisposed to significant anxiety, insomnia, risk-taking, or suicidal thinking, are candidates for pharmacotherapy in pregnancy. Women who have had severe episodes of depression that have recurred with medication discontinuation should also con- tinue pharmacotherapy. Consulting psychiatrists may be helpful in reassuring women about medications choices and providing or referring for adjunctive services. Full discussion of available research, treatment options, risks of undertreating depression, and benefits of medication are paramount in pregnancy. Medications should be used at the lowest dose necessary to improve symptoms, however, increased metabolism in pregnancy may necessitate higher doses relative to pre-pregnancy.

As stated, the selective serotonin reuptake inhibitors and the secondary amine tricyclic antidepressants are preferred agents in pregnancy and appear safe even in the first trimester. Personal or family history of response to a given agent frequently guides the first choice. Additionally, many women prefer to avoid the side effects of the tricyclic agents. The selective serotonin reuptake inhibitor fluoxetine has been best studied in pregnancy. Preliminary studies on sertraline, paroxetine, and citalopram are similarly encouraging without evidence of teratogenicity. These should be considered for women who have previously had good outcomes. The tricyclic antidepressants nortriptyline and desipramine are good candidates for use in pregnancy because they tend to cause less orthostatic hypotension, dry mouth and constipation. They should be considered in women who have failed trials with the SSRIs or with previous good response. Caution should be employed in women with severe suicidal ideation because of the risk of cardiac arrhythmias when tricyclics are used in overdose. Weaning tricylic antidepressants prior to the time of delivery is contraindicated because fetal withdrawal syndromes pose greater risk to the fetus than extrauterine withdrawal and places the woman at risk for relapse. MAOIs are contraindicated in pregnancy. There is limited information about pregnancy risks of bupropion, nefazodone, and venlafaxine. Generally patients should be seen by a physician or contacted by a member of the nursing staff every few weeks until symptoms begin to remit. Medications should be continued for 6 months following delivery to prevent exaccerbation during the postpartum. 


\subsection{Step 5 - adjunctive strategies for refractory illness}

Some women do not respond well to the first agent chosen. If a patient experiences partial symptom relief, generally increasing the dose of the primary agent is indicated. Choosing another agent within the SSRI or tricyclic class is indicated for patients who are entirely unresponsive to the first agent within the first trimester. Electroconvulsive therapy should be considered for severe debilitating depression with nutritional compromise. Referral for psychiatric co-management is advised in this circumstance. Disclosure of available research about use of these agents, and risk of undertreatment of illness is critical in preparing a woman and her family for use of the medications.

\subsection{Step $6-$ continuing or initiating treatment for depression during the postpartum}

Women who are at risk for postpartum depression include those with mood symptoms during pregnancy, marital discord, and family or personal history of depression. For women at risk but who remain asymptomatic, or those with mild symptoms of postpartum depression, nonpharmacologic prevention strategies (diet, exercise, stress management) may be employed initially. As in pregnancy, thyroid function testing and assessment of alcohol intake are important screens. Maintaining circadian rhythm is very important during the postpartum. Whenever possible, women at risk should arrange for doula services, or family assistance with night-time feedings. Women who have had prior episodes of severe postpartum depression should consider reintroduction of their antidepressant in the third trimester. This strategy ensures that they will have adequate blood levels upon delivery. For women with prior histories of mild illness, and those experiencing first episodes of postpartum depression, medications should begin after delivery. Many women choose to lactate and can be encouraged to do so, though for the severely depressed this may not be possible. Generally, the SSRIs as well as the tricyclic agents appear to be quite effective in postpartum depression and may be used during lactation. Benzodiazepines are relatively contraindicated in lactating women as they act as CNS depressants in the neonate and may cause kernicterus when used within the first week of life. Benadryl is a reasonable agent to consider for sleep, trazodone has been used clinically but is not well studied.

\section{Management of bipolar illness in pregnancy and postpartum}

\subsection{Step 1 - establishing a diagnosis}

Most patients who have bipolar illness will have been seen previously by a psychiatrist, and new onset bipolar illness should be referred for definitive diagnosis.

\subsection{Step $2-$ determining severity and course of illness}

When women have had long periods of quiescence in their illness prior to conception, a medication wean should be considered during the first trimester. Medications should be tapered gradually, to avoid relapse and should be done in consultation with psychiatric services. For women with prominent mood symptoms psychopharmacologic strategies are required.

\subsection{Step 3 - initiating pharmacotherapy}

In women with significant mood liability, insomnia, nutritional compromise or risk taking behavior, mood stabilizers should be used. They are relatively contraindicated in the first trimester, but symptom severity sometimes dictates continued use during this period. Discussion of risks of medications, including cardiac and neural tube defects, as well as risks of untreated illness, is paramount to encourage compliance with medications. Relapses of bipolar illness should be treated aggressively with mood stabilizers when clinically indicated. Exposure to the anticonvulsants in the first trimester is associated with an increased risk of neural tube defects. Therefore, lithium is pre- 
ferred in the first trimester because its risk for Ebstein's anomaly is relatively remote $(0.1 \%)$. Ultrasound with full fetal survey should be obtained at $16-18$ weeks to assess for congenital anomalies when any of the mood stabilizers are used. Folate (4 mg/day) is also recommended for all women 4 weeks prior to conception and throughout the first trimester when the anticonvulsants are used, pediatric teams should be arranged at the time of delivery to optimize neonatal outcomes. Lithium should be prescribed in multiple daily dosings to avoid exposing the infant to large variations in blood levels with close monitoring in the third trimester and postpartum due to variations in blood levels. It should generally be continued for 6-12 months following delivery to avoid postpartum exacerbation of illness.

\subsection{Step 4 - treatment resistance and treatment of psychosis}

ECT is indicated if rapid reconstitution is essential. Psychosis with delusions and command hallucinations present a psychiatric emergency, and it is this clinical situation which may be a predisposing factor in the disastrous consequence of suicide or fetal abuse. Hospitalization and the addition of neuroleptics are always required if the woman and her fetus are at risk.

\subsection{Step 5 - treatment of bipolar illness in postpartum}

All patients who have bipolar illness need to be monitored quite closely during the postpartum period. For patients who have had previous exacerbations of bipolar illness during the puerperium, it is prudent to re-institute the mood stablizer at the time of delivery, as this significantly reduces the risk of relapse from 50 to $70 \%$ to a more modest 10-20\%. Lithium levels should be closely monitored shortly after delivery because of the risk of toxicity. Breastfeeding is relatively contraindicated with use of lithium due to its concentration within the neonate. Carbamazepine, with its rapid metabolism is slightly preferred for nursing mothers, although these infants should be very closely monitored. Any evidence of suicidal thinking, hallucinations, voiced concern about harming the infant or disorganized thinking about the infant's health should be viewed as psychiatric emergencies. Hospitalization, aggressive pharmacotherapy and ECT are treatments of choice.

\section{Referral considerations}

Many obstetricians are quite comfortable managing uncomplicated major depression. Primary care is the 'de facto' mental health system in the country. Patients who respond well to SSRIs and tricyclic agents can usually be managed in primary care. Such patients should be monitored by physicians and nursing staff every few weeks until their acute phase symptoms (sleep, appetite, mood) have resolved. Compliance with medication is also important to monitor as over $50 \%$ of patients discontinue medication within the first month. Refractory illness or complex bipolar disorder may require psychiatric consultation. In women who have active suicidality, a history of psychosis, emerging symptoms of disorganized thinking, or hallucinations or delusions, referral for psychiatric management is recommended and hospitalization is often required. Other co-morbid problems (eating disorders, substance abuse, panic disorder with agoraphobia) or persistent psychosocial problems also frequently require more formalized mental health treatments.

\section{Conclusions}

All psychotropic agents freely cross the placental barrier and expose the fetus to those medications; therefore, the risks of untreated psychiatric illness must always be balanced with the risk of fetal or infant exposure. While some mood stabilizers may increase the absolute risk for teratogenicity, that risk should be considered in the entire context of the woman's illness and the adverse consequences of untreated depression.

Further study is essential to elucidate risk factors that predispose women to relapse with dis- 
continuation of medication. Additional information and long-term studies examining behavioral effects in children who are exposed to these agents in pregnancy and lactation are also essential as well as studies on newer agents and nutraceuticals.

The need for treatment of this serious, sometimes life-threatening, illness during the preand postpartum periods has been under-recognized with potentially serious consequences for women and their children. While more research is necessary to determine the fetal safety of newer antidepressant agents, much of the evidence to date indicates that there is no increased risk of teratogenicity or fetal anomalies with the use of antidepressant agents during pregnancy. New research and treatment guidelines will serve to inform the medical community about the appropriateness of treatment as well as state-of-the-art techniques and medications to serve this widespread and vulnerable patient population.

\section{References}

[1] Kessler RC, McGonagle KA, Nelson CB, Swartz M, Blazer DG. Sex and depression in the National Comorbidity Survey. II: Cohort effects. J Affect Dis 1999;30:15-26.

[2] Cohen LS, Heller VL, Rosenbaum JF. Treatment guidelines for psychotropic drug use in pregnancy. Psychosomatic 1989;30:25-33.

[3] Satcher D. Mental health: a report of the surgeon general. Department of Health and Human Services, 1999.

[4] Marcus SM, Barry KL, Flynn HA, Blow FC. Improving detection, prevention and treatment of depression and substance abuse in childbearing women: Critical variables in pregnancy and prepregnancy planning. University of Michigan Clinical Ventures Grant through Faculty Group Practice Pilot Data, 1998.

[5] Katon W, Von Korff M, Lin E, Bush T, Ormel J. Adequacy and duration of antidepressant treatment in primary care. Med Care 1992;30:67-76.

[6] Lin EH, Von Korff M, Katon W, Bush T, Simon GE, Walker $\mathrm{E}$ et al. The role of the primary care physician in patients' adherence to antidepressant therapy. Med Care 1995;33:67-74.

[7] Miller LJ. Clinical strategies for the use of psychotropic drugs during pregnancy. Psychiatry Med 1991;9:275-298.

[8] Burt VK, Hendrick FC. Concise guide to women's health. Washington, DC: AP Press, Inc, 1997.

[9] Kendell RE, Chalmers JC, Platz C. Epidemiology of puerpereal psychoses. Br J Psychiatry 1987;150:662-673.

[10] Altshuler LL, Cohen L, Szuba MP, Burt VK, Gitlin M, Mintz J. Pharmacologic management of psychiatric illness during pregnancy: dilemmas and guidelines. Am J Psychiatry 1996;153:592-606.

[11] Kulin NA, Pastuszak A, Sager SR, Schick-Boschetto B, Spivey G, Feldkamp M. Pregnancy outcome following maternal use of the new selective serotonin reuptake inhibitors: a prospective controlled multicenter study. J Am Med Assoc 1996;279:609-610.

[12] Patuszak A, Shick-Boschetto B, Zuber C, Feldkamp M, Pinelli M, Sihn S. Pregnancy outcome following firsttrimester. J Am Med Assoc 1993;269(17):2246-2248.

[13] Stowe ZN, Owens MJ, Landry JC, Kilts CD, Ely T, Llewellyn A. Sertraline and desmethylsertraline in human breast milk and nursing infants. Am J Psychiatry 1997; 154:1255-1260

[14] Nulman I, Rovert J, Stewart DE, Wolpin J, Gardner HA, Theis JG. Neurodevelopment of children exposed in utero to antidepressant drugs. $\mathrm{N}$ Engl $\mathrm{J}$ Med 1997;336(4):258-262.

[15] Ericson A, Kallen B, Wiholm B. Delivery outcome after the use of antidepressants in early pregnancy. $\mathrm{N}$ Engl J Med 1996;55:503-508.

[16] Chambers CD, Johnson KS, Dick LM, Felix RJ, Jones KL. Birth outcomes in pregnant women taking fluoxetine. N Engl J Med 1996;335(14):1010-1015.

[17] Robert E. Treatment depression in pregnancy. N Engl J Med 1996;335(14):1056-1058.

[18] Glaxo Welcome. Bupropion pregnancy registry interim report. Research Triangle Park, NC, 1999.

[19] Cohen LS, Friedman JM, Jefferson JW, Johnson EM, Weiner ML. A reevaluation of risk of in utero exposure to lithium. J Am Med Assoc 1994.

[20] Scolnik D, Nulman I, Rovert J, Gladstone D, Czuchta $\mathrm{D}$, Gardner HA et al. Neurodevelopment of children exposed to phenytoin and carbamazepine monotherapy. J Am Med Assoc 1996;271:767-770.

[21] Diagnostic and Statistical Manual of Mental Disorders, 4th ed. (DSM-IV) American Psychiatric Association: Washington, DC, 1994. 